\title{
Estrogen receptor alpha deletion enhances the metastatic phenotype of Ron overexpressing mammary tumors in mice
}

\author{
Aaron M Marshall ${ }^{1 \dagger}$, Rebecca J McClaine ${ }^{2 \dagger}$, Devikala Gurusamy ${ }^{1}$, Jerilyn K Gray ${ }^{1}$, Kara E Lewnard ${ }^{1}$, Sohaib A Khan ${ }^{1}$ \\ and Susan E Waltz ${ }^{1,3^{*}}$
}

\begin{abstract}
Background: The receptor tyrosine kinase family includes many transmembrane proteins with diverse physiological and pathophysiological functions. The involvement of tyrosine kinase signaling in promoting a more aggressive tumor phenotype within the context of chemotherapeutic evasion is gaining recognition. The Ron receptor is a tyrosine kinase receptor that has been implicated in the progression of breast cancer and evasion of tamoxifen therapy.

Results: Here, we report that Ron expression is correlated with in situ, estrogen receptor alpha (ER $\alpha$ )-positive tumors, and is higher in breast tumors following neoadjuvant tamoxifen therapy. We also demonstrate that the majority of mammary tumors isolated from transgenic mice with mammary specific-Ron overexpression (MMTVRon mice), exhibit appreciable ER expression. Moreover, genetic-ablation of ER $\alpha$, in the context of Ron overexpression, leads to delayed mammary tumor initiation and growth, but also results in an increased metastasis.

Conclusions: Ron receptor overexpression is associated with ER $\alpha$-positive human and murine breast tumors. In addition, loss of ERo on a Ron overexpressing background in mice leads to the development of breast tumors which grow slower but which exhibit more metastasis and suggests that targeting of ERo, as in the case of tamoxifen therapy, may reduce the growth of Ron overexpressing breast cancers but may cause these tumors to be more metastatic.
\end{abstract}

Keywords: Ron Receptor, MST1R, Hepatocyte growth factor-like protein, breast cancer, estrogen receptor

\section{Background}

To date, the most successful pharmacological therapies specifically targeting breast cancer include anti-estrogens and receptor tyrosine kinase (RTK) modulating drugs [1]. Accordingly, there have been numerous studies examining signaling paradigms between estrogen and RTK signaling pathways [2-4] which have provided evidence that RTKs are able to activate estrogen receptor alpha $(E R \alpha)$ in breast cancers independent of its ligand estrogen. This activation of ER $\alpha$ by RTKs leads to an $E R \alpha$ transcriptional program that enhances cell survival. The dependency of this activation on the RTK ligand is

\footnotetext{
* Correspondence: susan.waltz@uc.edu

+ Contributed equally

'Department of Cancer and Cell Biology, University of Cincinnati, College of Medicine, Cincinnati, OH 45267-0521, USA

Full list of author information is available at the end of the article
}

still an area of active investigation. More importantly, however, this signaling crosstalk between RTKs and ER $\alpha$ may predict resistance to anti-estrogen hormonal therapies, including tamoxifen [2,5]. Specifically, studies have shown that activation of EGFR, Her2, cMet, IGFR, RET and recently, Ron RTK, lead to phosphorylation and activation of ER $\alpha$ which enhances survival of breast cancer in the presence of anti-estrogen therapy [4,6-8].

Ron is a cell surface RTK related to the c-Met receptor that has been identified as an oncogene in the development and growth of human epithelial tumors [9]. In the developing mammary gland, Ron is expressed during the pubertal growth stages, and then again during pregnancy and lactation, and its expression remains low in quiescent glands [10]. In normal mammary development, genetic loss of Ron signaling has been shown to
C Biomed Central 
alter mammary gland branching morphogenesis during puberty [10]. In cell lines, wild-type Ron overexpression is associated with induction of oncogenic properties, including malignant transformation, proliferation, and migration [11]. Overexpression of Ron in transgenic mouse models of both lung and breast cancer is associated with tumorigenesis in both organs [12,13]; while deletion of Ron in transgenic mice expressing polyoma virus middle $\mathrm{T}$ antigen caused a significant reduction in breast tumor formation and growth [14]. Additionally, Ron is known to be upregulated in a number of human epithelial cancers, including breast, lung, stomach, colon, pancreas, and prostate. Specifically, Ron is highly expressed in approximately $50 \%$ of human breast cancers [15]. Given the important role of Ron in human and mouse tumorigenesis, identifying the functions and signaling pathways associated with this RTK may provide essential clues to combat disease progression.

Recent data has shown that Ron activation leads to the phosphorylation and activation ER $\alpha$. In this case, exogenous overexpression of Ron or ligand activation of endogenous Ron led to enhanced survival of several $E R \alpha$-positive breast cancer cell lines in the presence of the anti-estrogen therapy tamoxifen [4]. Estrogen receptor alpha and its ligand estrogen are important regulators of mammary gland development and breast carcinogenesis. During development ER $\alpha$ is critical for peripubertal ductal elongation, and is a permissive factor in alveolar expansion during pregnancy [16-19]. In cancer, ER $\alpha$ transcriptional programming provides survival and growth stimulation that is advantageous for tumors and the majority of human breast cancers express ER $\alpha$. Consequently, ER $\alpha$ is the target of a family of anti-estrogen pharmacological compounds including tamoxifen. Although tamoxifen treatment is often initially successful at preventing the proliferative effect of estrogen on $\mathrm{ER} \alpha$, the preponderance of patients develop tamoxifenresistance [5] and the recurrent tumors tend to be more aggressive [17]. Tamoxifen resistance has become a major obstacle to effective treatment of ER $\alpha$-positive breast cancer.

Complementing previously published work, we show here that Ron is overexpressed and correlated with early stage ER $\alpha$-positive breast cancers. To directly demonstrate the effect of ER $\alpha$ inactivation has on breast cancer, in the presence of Ron, we have created ER $\alpha$ -replete and ER $\alpha$-conditionally deficient animals on a Ron overexpressing background. We show for the first time that mammary-specific ER $\alpha$ deletion increases breast tumor latency, but also leads to a more metastatic phenotype. Interestingly, these studies suggest a negative consequence of anti-estrogen therapy in Ron expressing tumors on the promotion of metastasis.

\section{Results}

Correlations between MST1R/Ron, ER \& Breast Cancer Staging

Previous studies have shown that Ron activation leads to phosphorylation and activation of ER [4]. However, no studies have shown an association between Ron and ER in the human patient population. To accomplish this we first mined publically available data in Oncomine. Two independent microarray studies demonstrated that Ron (MST1R) expression positively correlates with ER-positive breast cancers (Figure 1A) [20,21]. Furthermore, Ron expression is also increased in patients following neoadjuvant tamoxifen therapy (Figure 1B).
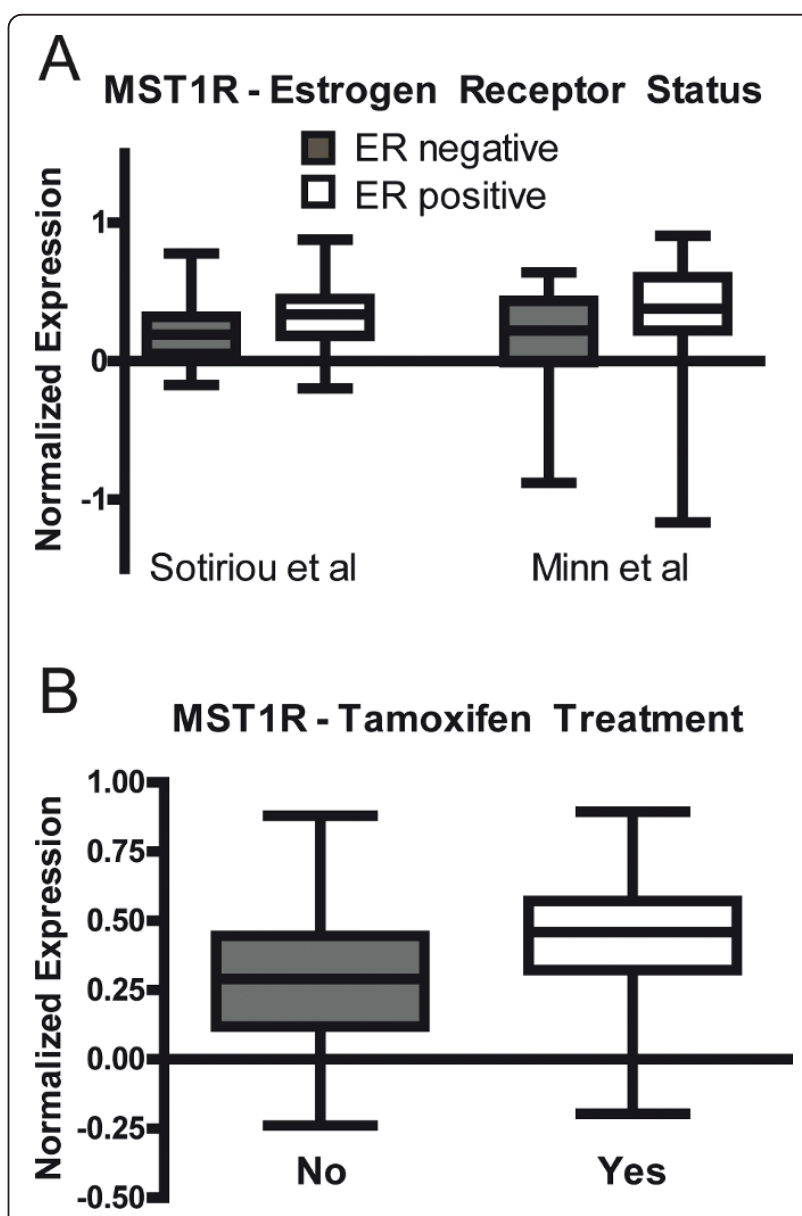

Figure 1 MST1R (Ron) mRNA expression in publically available data sets. (A) Comparison of MST1R expression in human breast tumor tissue between estrogen receptor positive and estrogen receptor negative tumors. Shown are box and whisker plots from two

independent data sets; line represents the median, box represents interquartile range and whiskers represent $\mathrm{min} / \max (p<0.01$ for each comparison utilizing the Mann-Whitney test). (B) Utilizing the Sotiriou et al. data set, we show MST1R expression in breast cancer tissue between patients who received neoadjuvant tamoxifen (Yes) versus those who did not receive neoadjuvant therapy (No) $(p<0.01$, Mann-Whitney test). 
Ron receptor overexpression has been reported in approximately half of breast cancers [15,22]. However, little information is known regarding the association of Ron overexpression with a particular subtype or stage of human cancer. To address this, immunohistochemistry for Ron was performed on three independent breast cancer tissue arrays. The arrays contained 250 human breast tumor specimens. Ron staining and expression were determined as previously described [22,23]. As depicted in Figure 2A, Ron was found to be more highly expressed in carcinomas in situ versus invasive carcinomas. In an analysis of Ron expression by T-stage, Figure 2B demonstrates that Ron is inversely associated with T-stage with Ron expression being highest in the in situ tumors (Tis), especially when compared to T2 and T3/4.
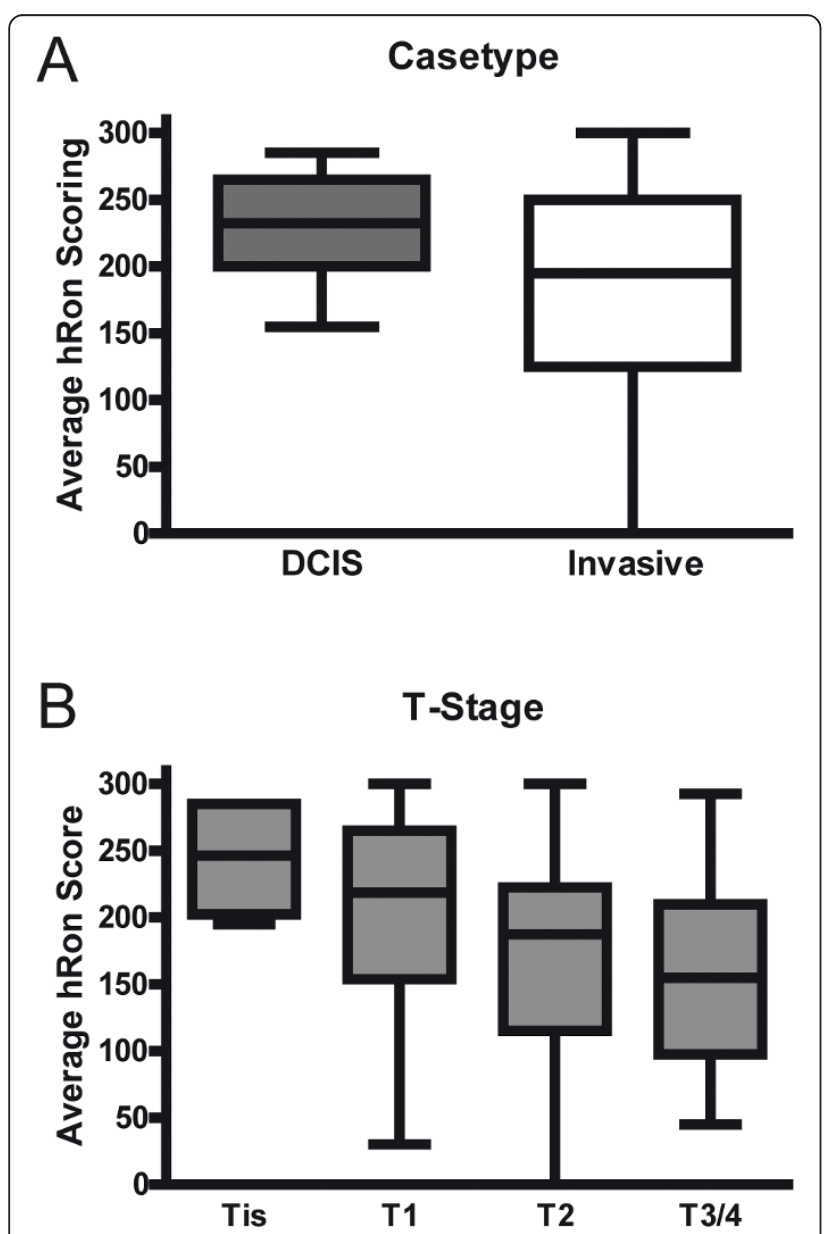

Figure 2 Tissue array immunohistochemical staining for Ron. (A) Comparison of Ron staining score in DCIS versus invasive cancer subtypes ( $p<0.01$, Mann-Whitney test, $n=250$ ). (B) Comparison of Ron staining score among T-stage (Tis = carcinoma in situ, T1 = tumor $<20 \mathrm{~mm}, \mathrm{~T} 2=$ tumor $>20 \mathrm{~mm}$ and $<50 \mathrm{~mm}$ and $\mathrm{T} 3 / 4=$ tumor $>50 \mathrm{~mm}$ ). Tis versus T1, and T2 versus T3/4 did not reach statistical significance, but all other pairwise comparisons had $\mathrm{p}$ values $<0.05$ by Kruskal-Wallis test followed by Dunn's multiple comparisons $(n=250)$.
T3 and T4 were grouped together due to the small number of T4 tumors present on the array. Tis versus $\mathrm{T} 1$, and $\mathrm{T} 2$ versus $\mathrm{T} 3 / 4$ did not reach statistical significance, but all other pairwise comparisons had p-values $<0.05$. Altogether, the data suggests Ron is prominently featured in early stage ER+ tumors.

\section{Tumor Characterization in WAP-Cre/ER ${ }^{\mathrm{fl} / \mathrm{fl}} / \mathrm{MMTV}-$ Ron Mice} Since Ron is associated with early stage ER+ tumors, a set of genetic experiments was performed to test the hypothesis that tumor progression is altered in mice where ER $\alpha$ is non-functional and Ron is overexpressed. To accomplish this, the MMTV-Ron driven mouse model of breast cancer was utilized. As previously reported, MMTV-Ron mice develop breast cancer with $100 \%$ penetrance [13]. To generate $\mathrm{ER}^{\mathrm{fl} / \mathrm{fl}} / \mathrm{MMTV}$-Ron mice (ERRN), MMTV-Ron mice were crossed to $E R^{\mathrm{fl} / \mathrm{fl}}$ mice. To create mice on the MMTV-Ron background that are deficient in ER $\alpha$ protein, we then crossed ERRN mice with transgenic mice expressing Cre recombinase under the direction of the whey acidic protein promoter (WAP-Cre) to obtain WAP-Cre Mice/ER ${ }^{\mathrm{fl} / \mathrm{fl}}$ / MMTV-Ron (WPERRN) mice and littermate controls (ERRN). The ERRN control mice develop mammary tumors with $100 \%$ penetrance and a median time to palpable tumor of 314 days (Figure 3A). The WAP-Cre Mice/ER ${ }^{\mathrm{fl} / \mathrm{fl}} / \mathrm{MMTV}$-Ron (WPERRN) mice also develop mammary tumors with $100 \%$ penetrance; however the WPERRN mice exhibited a significant increase in tumor latency ( $\mathrm{p}<0.05$, log-rank test) compared to controls (Figure 3A). The median tumor latency in this group is increased by 12.5 days to 326.5 days compared to mammary tumors from ERRN mice.

To verify Cre-mediated deletion of a 680 bp region of the ER $\alpha$ allele, conventional PCR was performed on DNA isolated from excised mammary tumors of ERRN and WPERRN mice. All ERRN mice had the endogenous full length 1280 bp wild-type ER $\alpha$ allele, represented by lanes 1 and 2 in Figure 3B. Of the 16 mammary tumors analyzed from WPERRN mice, all had both the wild-type ER $\alpha$ allele (1280 bp band) and the knockout ER $\alpha$ allele (600 bp band). Four animals had a wild-type band that was greater than 2 -fold stronger than the knockout band as shown in lane 5 (Figure 3B) suggesting limited $\mathrm{ER} \alpha$ deletion in this tumor. These mice were excluded from all analyses, including Figure 3A. The remaining 12 tumors had a greater than 5-fold more intense knockout band compared to the wild-type band and are represented by lanes 3-4, 6-7 (Figure 3B). To determine the extent of $E R \alpha$ protein depletion, whole tissue lysates from ERRN and WPERRN mammary tumors were examined by Western analyses. ER $\alpha$ protein was reduced approximately $50 \%$ as shown in Figure $3 \mathrm{C}$ and quantified in 


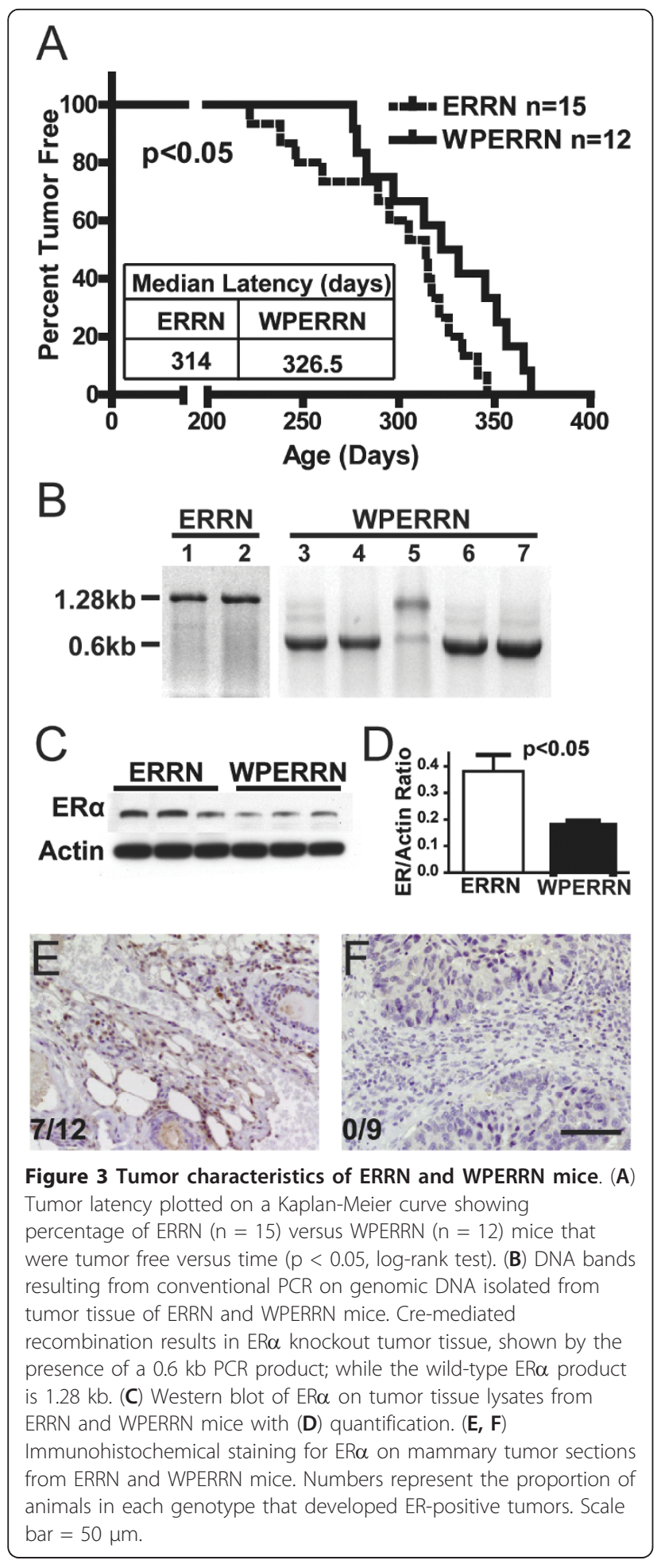

Figure 3D. Given the heterogeneous origin of the mammary tumor tissue, and the expression pattern of whey acidic protein, it is not surprising that ER $\alpha$ is still present, albeit at lower levels. To verify that ER $\alpha$ was indeed deleted from the tumors of WPERRN mice, and to document the percentage of ERRN mice that developed ER $\alpha$-positive tumors, we performed immunohistochemistry on tumor sections. Using a scoring system previously described for determining ER-positivity [24], we determined that in ERRN mice, ER $\alpha$-positive tumors occur approximately $58 \%$ of the time (7/12) (Figure 3E). In contrast, none of the nine WPERRN mice had ER $\alpha$-positive tumors (Figure 3F).

\section{Analysis of proliferation and death rates in WPERRN and ERRN tumors}

The increase in latency of WPERRN tumors compared with ERRN tumors suggests that tumor cell proliferation and/or apoptosis may be altered. To examine this, we performed BrDU and TUNEL staining on end-stage mammary tumors, which was defined as a primary tumor reaching $\sim 2.5 \mathrm{~cm}^{3}$ in size. Interestingly, BrDU incorporation was not significantly different in WPERRN mice versus ERRN mice (Figure 4A), nor was TUNEL staining (Figure 4B). To more accurately assess the proliferation and apoptosis rates during tumor initiation, we harvested mammary glands from age matched WPERRN and ERRN mice at 220 days of age and stained for BrDU and TUNEL. This time point was determined to be optimal for obtaining mammary glands with hyperplasia, but prior to palpable tumor formation. At 220 days old, WPERRN mammary epithelial cells displayed significantly lower BrDU incorporation than in ERRN mice (Figure 4C). Representative images for 220 day old BrDU staining are shown in Figure $4 \mathrm{E}$ and $4 \mathrm{~F}$. TUNEL staining at 220 days old was low in these neoplastic regions and was not different between ERRN and WPERRN animals (Figure 4D, G and 4H).

\section{Alterations in metastatic burden in WAP-Cre/ER ${ }^{\mathrm{fl} / \mathrm{fl}} / \mathrm{MMTV}$ - Ron Mice}

The liver and lungs were targeted for metastatic foci examination and quantification. Previously, we reported that MMTV-Ron transgenic mice develop metastases to these two organs [13]. The lungs and livers were excised and fixed in formalin at the time of sacrifice. The lungs of ERRN and WPERRN mice both contained metastases that were histologically similar (Figure 5A). To quantify the overall metastatic burden, we first determined the metastatic rate to the lungs using a binary system (+ or -). The proportion of mice with metastases between ERRN and WPERRN mice was not statistically different $(89 \%$ and $96 \%$ respectively; $Z$ value $=0.39)$ (Figure $5 B)$. However, histological examination of the number of metastatic foci in a single lobe revealed that the WPERRN mice carried a higher metastatic burden in the lungs than the ERRN mice (Figure 5C). Immunohistochemical staining for cytokeratin 18 verified the origin 


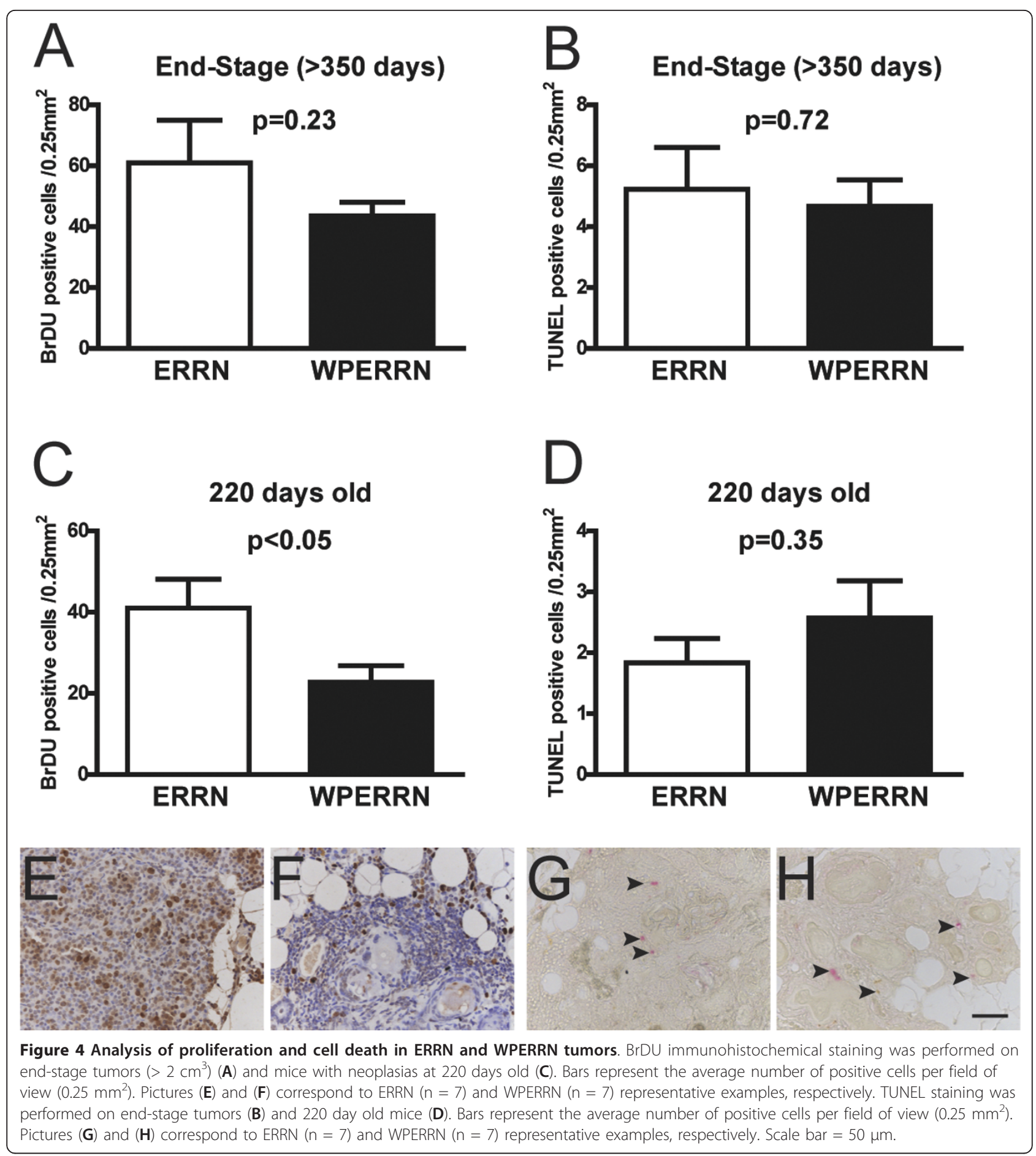

of the metastases as from the breast (data not shown). Next we examined the livers of ERRN and WPERRN mice. As with the lungs, both genotypes had liver metastases that were histologically indistinguishable from each other (Figure 5D). Again using a binary scoring system for the presence of liver metastases, we determined that $56 \%$ of ERRN mice and $96 \%$ of WPERRN mice had metastatic liver foci $(\mathrm{P}<0.01 \mathrm{Z}$ value $=2.98)$ (Figure $5 \mathrm{E})$. We confirmed the origin of the foci by immunostaining for cytokeratin 5, which was determined to be present in the MMTV-Ron mammary tumors and not in hepatocytes (data not shown). Next, we sought to determine the extent of ER positivity in the metastases. The metastases in all genotypes and both tissues stained 


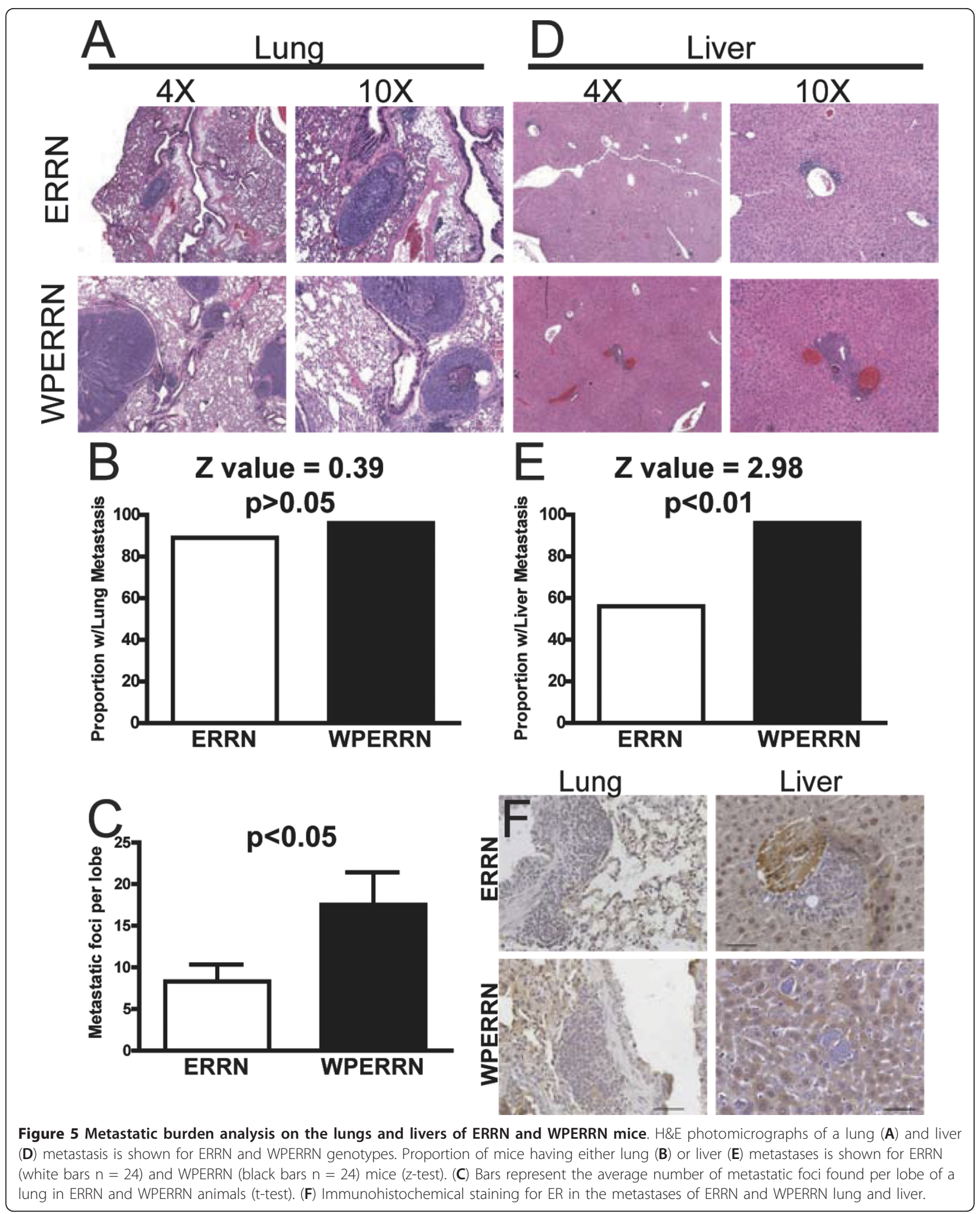


negative for ER (Figure 5F). This reduction in the number of metastases may be reflective of the proportion of ER negative tumors in the different genotypes. Overall, we demonstrate the MMTV-Ron driven tumors that have deleted ER $\alpha$ have a higher metastatic burden to the lungs and liver.

$E R \alpha$ deletion in MMTV-Ron tumors is associated with decreased expression of ER-dependent genes

To explore a potential mechanism by which ER $\alpha$ deletion leads to increased latency and enhanced metastasis, we evaluated the expression of ER $\alpha$-dependent target genes by real-time PCR and western blotting. Cyclin D1 (CCND1), mothers against decapentaplegic homolog 3 (SMAD3) and cathepsin D (CTSD) are genes known to be stimulated by ER $\alpha$ activation [25-27]. Tumor tissue representing WPERRN and ERRN mice was homogenized and the total RNA isolated. Real-time PCR was performed on seven independent samples, plated in duplicate. In WPERRN mice the expression of CCND1, SMAD3 and CTSD were all decreased compared to ERRN animals by approximately $60 \%$ ( $\mathrm{P}<0.01,0.02,0.005$ respectively) (Figure 6A, B \& 6C respectively). Whole protein lysates from WPERRN and ERRN tumors were also analyzed for expression of cyclinD1. Expression of cyclinD1 was decreased in WPERRN tumors, which correlates with the gene expression studies (Figure 6D). Furthermore, progesterone receptor, a well-known protein product of ER signaling was also decreased to a similar extent as cyclinD1 (Figure 6D).
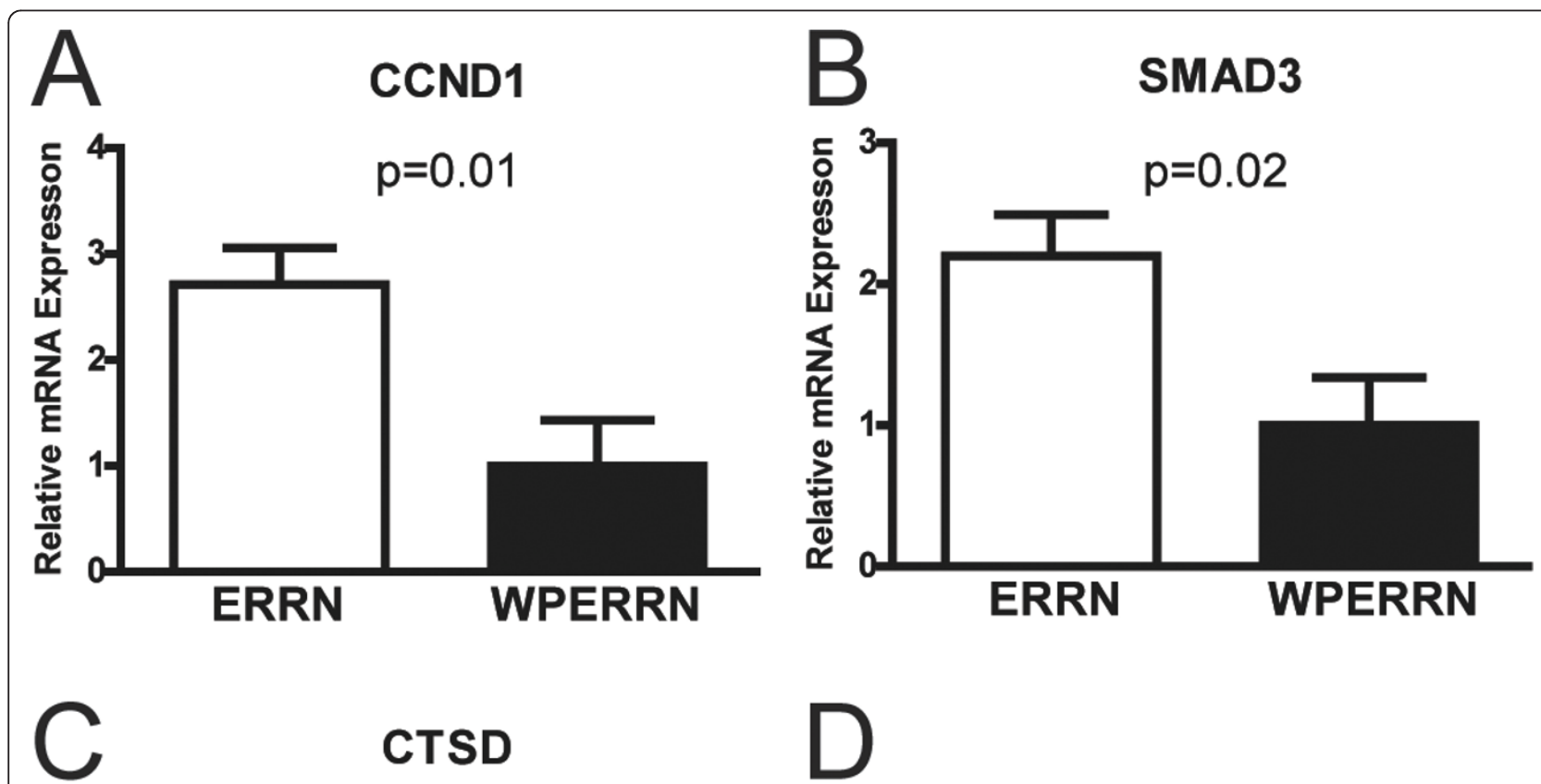

CTSD
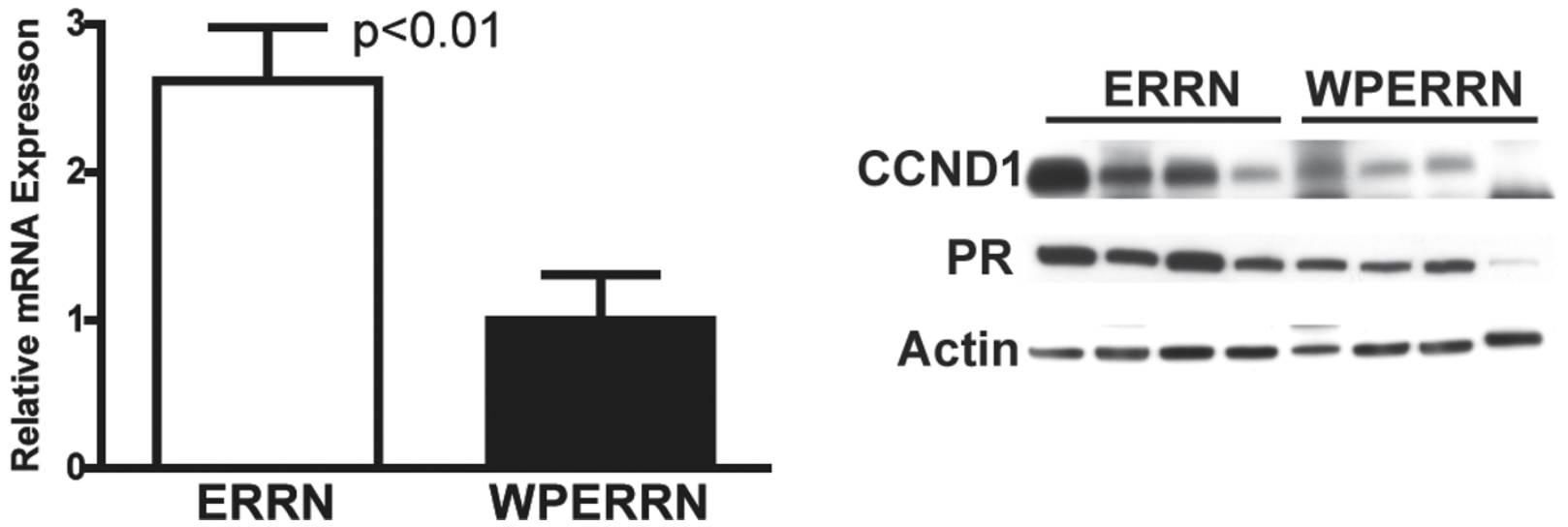

Figure 6 Expression changes of ER-regulated genes. (A, B, C) Bars represent the relative mRNA expression levels between ERRN $(n=7)$ and WPERRN $(n=7)$ tumor lysates, quantified by qPCR. The ER-regulated genes $(\mathbf{A})$ CyclinD1 (CCND1) (B) SMAD3 and (C) cathepsin D (CTSD) were analyzed (t-test). (D) Protein lysates were also analyzed by Western blotting for expression of cyclin D1 and progesterone receptor. 


\section{Pure $\mathrm{ER}^{\mathrm{fl} / \mathrm{fl}} \mathrm{Ron}$ and $\mathrm{ER}^{-/-}$Ron cells recapitulate tumor growth phenotypes}

Although, WPERRN animals had delayed tumor latency, the ER $\alpha$ deletion did not display $100 \%$ penetrance (see Figure $3 \mathrm{~B}$ ). Therefore to confirm the tumor growth phenotype, we established a cell line derived from an $\mathrm{ER}^{\mathrm{fl} /}$ ${ }^{\mathrm{fl}}$ MMTV-Ron tumor. We subsequently infected the $\mathrm{ER}^{\mathrm{fl} /}$ ${ }^{\mathrm{fl}}$ MMTV-Ron tumor cells with an adenovirus expressing Cre-recombinase and GFP or GFP alone. GFP-positive cells were isolated by FACS and cultured to establish $E R^{\mathrm{fl} / \mathrm{fl}}$ Ron cells and $\mathrm{ER}^{-/-}$Ron cells. PCR analysis confirmed the Cre-mediated deletion of a $600 \mathrm{bp}$ region of exon 3 of ER $\alpha$ (Figure 7A). This deletion was still evident at passage 23, with no contaminating wild-type ER $\alpha$ allele detected (data not shown); and all subsequent experiments were performed between passages 5 and 15 . First, the proliferative rates of $E R^{\mathrm{fl} / \mathrm{fl}}$ Ron cells and $\mathrm{ER}^{-/ \text {- }}$ Ron cells were compared by the MTT assay and BrDU incorporation. Confirming the phenotype of ERRN and WPERRN mice, the $E R^{\mathrm{fl} / \mathrm{fl}}$ Ron cells displayed an increased proliferative rate compared to $\mathrm{ER}^{-/-}$Ron cells, by both MTT (Figure 7B) and BrDU incorporation experiments (Figure 7C). No differences in cell death or apoptosis were observed between the $E R^{\mathrm{fl} / \mathrm{fl}}$ Ron and $\mathrm{ER}^{-/}$Ron cells as judged by Annexin $\mathrm{V}$ and propidium iodide (PI) staining (data not shown). Next, to detect differences in metastatic potential, migration experiments were performed. Again supporting the phenotype observed in mice, the $\mathrm{ER}^{-1}$ Ron cells migrated more aggressively across a transwell membrane (Figure 7D). The $\mathrm{ER}^{-1-}$ Ron cells also invaded significantly more on transwell membranes which were coated with Matrigel ${ }^{\circledR}$, a basement membrane protein (data not shown). Furthermore, when these same cells were injected into the mammary fat pads of adult athymic nude mice, larger tumors were observed in $\mathrm{ER}^{\mathrm{fl} / \mathrm{fl}}$ Ron cell orthotopic injections compared to $\mathrm{ER}^{-/-}$Ron cells (10 weeks) (Figure 7E \&7F). Metastases were not found in the lungs or livers of either group of nude mice at the time of sacrifice; prohibiting analysis of metastatic burden.

\section{Discussion}

In this study we have genetically deleted ER $\alpha$ in a relevant model of murine mammary cancer (MMTV-Ron). We have shown that Ron expression is associated with early stage ER+ human breast cancers with the highest levels of Ron observed in carcinomas in situ. Ductal carcinomas in situ (DCIS) represent an increasing population of new patient diagnoses. Currently, 20-30\% of new cases are classified in this type [28] and evidence exists that DCIS is a precursor lesion for invasive cancer [28]. Therapeutic options for treating DCIS depend on hormone status and the patient's wishes. Patients with ER+ DCIS usually undergo lumpectomy and receive tamoxifen as a neoadjuvant or adjuvant therapy [28]. Since Ron is often overexpressed in these types of tumors, and Ron has previously been shown to mediate tamoxifen insensitivity, our data suggest that Ron may be an important biomarker for predicting chemotherapeutic resistance.

Previous work has shown that estrogen acts as a carcinogen via three independent mechanisms: (1) stimulation of proliferation through receptor signaling, (2) direct genotoxic effects via cytochrome P450, and (3) induction of aneuploidy [29]. In addition, the role of estrogen in stimulating proliferation in both normal and neoplastic tissue is well documented (30). Proliferation due to estrogen is dependent on ER $\alpha$. In this study, we were able to remove estrogen-ER $\alpha$ mediated proliferation by genetic manipulation. During tumor development, we observed that ER $\alpha$ deficient mammary tumors have a lower proliferative rate as measured by BrDU incorporation. Our studies also document an increase in tumor latency in these mice. Furthermore, cells derived from these tumors and manipulated to re-create the ERreplete and ER-knockout conditions recapitulated the proliferative phenotype observed in these animals. This confirms that in our MMTV-Ron driven model of carcinogenesis, estrogen-ER $\alpha$ signaling plays a proliferative role that can be attenuated by ER $\alpha$ deletion.

Interestingly, our studies provide important information related to the loss of ER $\alpha$ in the context of Ron overexpression, in that while mammary tumors with this loss grew slower, the tumors were overall more metastatic. This data suggest that the context in which ER $\alpha$ is deleted (or inhibited as in the case of anti-estrogen therapy akin to clinical studies), Ron may play an important role in regulating tumor progression and metastasis. In this case, a suggestion of dually targeting Ron and ER $\alpha$ may prove advantageous. While further studies are warranted to understand the mechanisms associated with the enhanced metastatic phenotype, a potential mechanism is that in WPERRN tumors compared to controls, the levels of the ER-regulated genes SMAD3 and cathepsin D are lower. Decreased expression of SMAD3 has been shown to uncouple TGF-beta signaling, resulting in a local immunosuppressive environment [30]. Cathepsin D functions primarily as a lysosomal peptidase in the processing of antigen for presentation. Inhibition or decreased expression of cathepsin D results in decreased antigen processing and presentation [31]. In conjunction with ER-dependent gene changes, Ron activation has been shown to play an important role in the suppression of innate immunity $[32,33]$. Taken altogether, the decreased expression of SMAD3 and cathepsin D due to ER loss, and concomitant overexpression of Ron may provide an environment favoring immune system evasion, which may be 


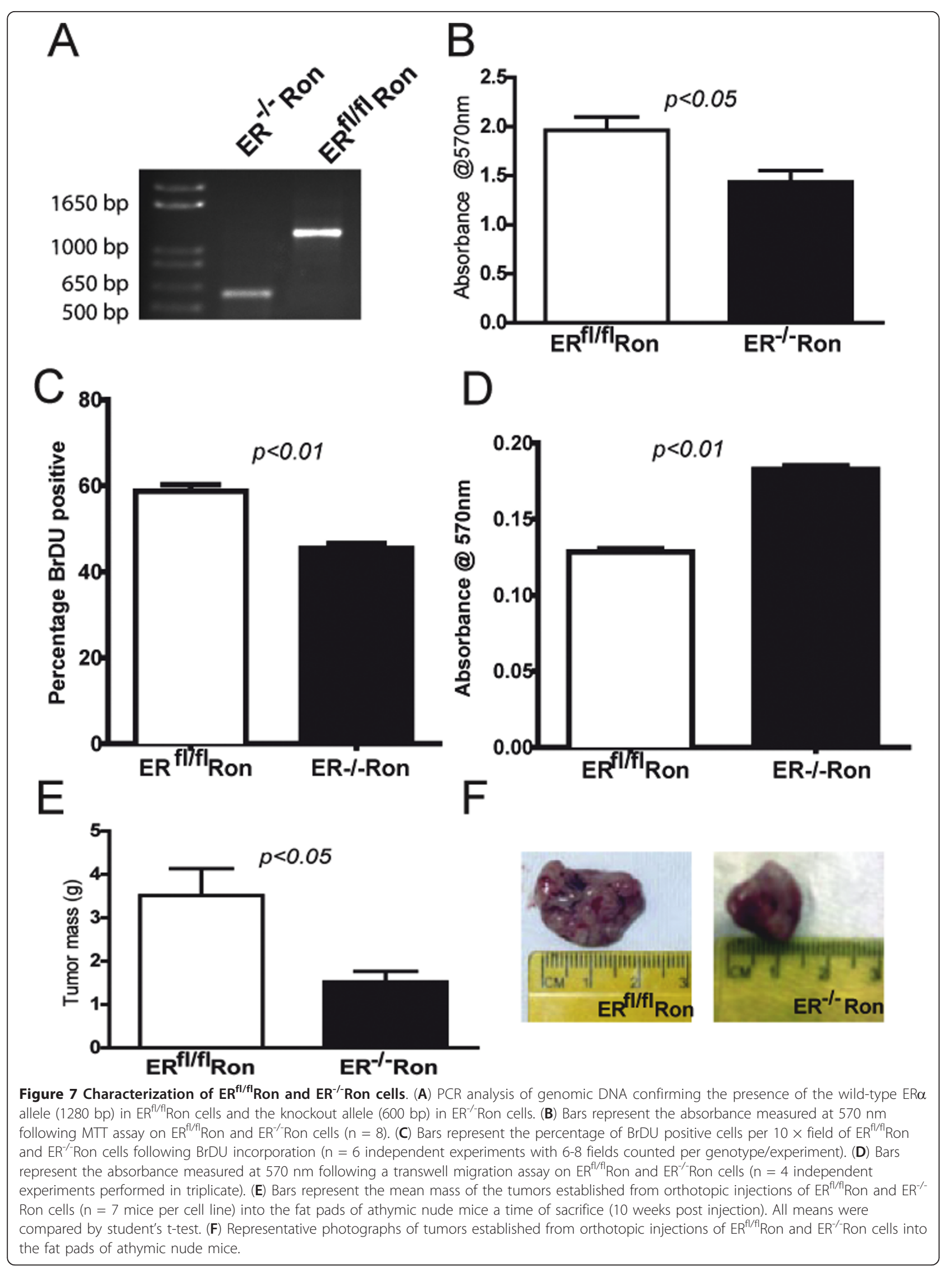


contributing to the enhanced metastatic phenotype. Testing this hypothesis, preliminary experiments showed that F480 staining in primary tumors was not significantly different between ERRN and WPERRN genotypes (data not shown). Further investigation is needed to verify and expand upon these findings.

The increased metastatic burden found in the WPERRN mice is interesting on two levels. First, these mice had neoplastic lesions that were slower to proliferate than ERRN neoplasias. There has been recent evidence suggesting that slower growing tumors metastasize at a higher rate [34]. The second level where the alteration in metastasis is interesting is with respect to anti-estrogen endocrine therapies. Tamoxifen and other anti-estrogen therapies ultimately fail due to acquired resistance. The endocrine therapy selects for tumor cells that can survive without the proliferative stimulation from estrogen-ER $\alpha$ activation. One observed consequence of resistance is increased tumor aggressiveness [35]. Our studies suggest that similar mechanisms are occurring in the WPERRN mice, where ER $\alpha$ has been deleted, rather than pharmacologically antagonized. In short, this model genetically recapitulates some aspects of acquired chemotherapeutic resistance due to ER inhibition, namely less proliferative tumors with an increased metastatic capacity.

\section{Conclusions}

In summary, the data herein demonstrate that Ron is overexpressed breast cancers with high levels or Ron observed in early stage ER $\alpha$-positive breast cancers. Our studies also show that a conditional loss of ER $\alpha$ in mammary epithelium of Ron overexpressing mice leads to the development of breast tumors which exhibit diminished growth and increased metastases.

\section{Methods}

\section{Animal Procedures}

MMTV-Ron $(\mathrm{FVB} / \mathrm{N})$ mice were crossed to $\mathrm{ER} \alpha^{\mathrm{fl} / \mathrm{fl}}$ (C57/B6) mice to generate ER ${ }^{\mathrm{fl} / \mathrm{fl}} / \mathrm{MMTV}$-Ron animals, abbreviated ERRN. Both transgenic strains are independently described elsewhere $[13,18]$. The ERRN mice were then crossed with transgenic mice carrying an allele for whey acidic protein promoter driving expression of Cre recombinase (WAP-Cre) $[36,37]$ to create WAP-Cre/ERa $\alpha^{\text {fl/fl }} /$ MMTV-Ron animals, abbreviated WPERRN, plus littermate controls. Primers used for genotyping and recombination efficiency were described previously $[13,18]$. For tumor production and to ensure WAP-Cre expression, all females used in experiments were housed with males throughout the duration of the experiment to promote parity. All females were multiparous, and no differences in parity were observed between WPERRN and ERRN animals. Mice were palpated bi-weekly for determination of tumor latency. Tumors reaching $20 \%$ of body mass were considered end-stage, and animals were sacrificed. Athymic nude mice were purchased from NCI-Frederick (Frederick, $\mathrm{MD})$. For orthotopic injections, $2 \times 10^{6}$ cells were suspended in a 1:1 mixture of DMEM:F12 and Matrigel ${ }^{\mathbb{B}}$ (BD Biosciences) and injected into the fat pad of anesthetized mice. All procedures were approved by the University of Cincinnati Institutional Animal Care and Use Committee.

\section{Reagents}

Primers used for genotyping mice were as follows: MMTV-Ron forward 5'TGG GTG GTG AGG TCT GCC AAC ATG A3', reverse 5'CCG TCT TCG GGA GTT AAA GAT CAG GG3'; ER $\alpha^{\mathrm{fl} / \mathrm{fl}}$ forward 5'TGG GTT GCC CGA TAA CAA TAA C3', reverse 5'AAG AGA TGT AGG GCG GGA AAA G3'; WAP-Cre forward 5'CAT CAC TCG TTG CAT CGA CC3', reverse 5'TAG AGC TGT GCC AGC CTC TTC3'. The ER $\alpha^{\text {fl/fl }}$ primers were also used for determining the deletion event in Cre-positive tumors and Cre-infected cells. Adenovirus expressing Cre and GFP or GFP described previously [22]. MTT reagent (Sigma, St. Louis, MO) was dissolved in PBS, added to the cells for 3 hours and the intensity of the resulting solution quantified on spectrophotometer at $570 \mathrm{~nm}$.

\section{Immunoblotting and Immunohistochemistry}

For immunoblotting, whole cell lysates were prepared in radioimmunoprecipitation assay (RIPA) buffer $(20 \mathrm{mM}$ Tris, $150 \mathrm{mM}$ sodium chloride, $2 \mathrm{mM}$ EDTA, $0.1 \%$ SDS, $1 \%$ Triton-X 100, 0.5\% sodium deoxycholate, $10 \%$ glycerol) supplemented with Complete protease inhibitor tablets (Roche, Palo Alto, CA) and HALT phosphatase inhibitors (Pierce, Rockford, IL). Protein concentrations were determined by Micro BCA Protein Assays (Pierce, Rockford, IL). Lysates were then boiled for 10 minutes in buffer containing beta-mercaptoethanol and separated by SDS-PAGE. Immunoblotting was carried out according to standard procedures on PVDF membrane, with enhanced chemiluminescence detection (GE Healthcare, Piscataway, NJ). Antibodies used were the following: anti-mouse ER $\alpha$ (clone MC-20; Santa Cruz, Santa Cruz, CA), anti-human Ron $\beta$ (clone C-20 Santa Cruz, Santa Cruz, CA), anti-mouse progesterone receptor (clone C-19, Santa Cruz), and anti-mouse cyclinD1 (clone 72-13G, Santa Cruz). Densitometry was performed on NIH ImageJ analysis software. For immunohistochemistry, tissues were fixed in neutral-buffered formalin, embedded in paraffin, and sectioned. Rehydrated sections were subjected to antigen retrieval and incubated in primary antibody overnight at $4^{\circ} \mathrm{C}$. Sections were then, washed, incubated in biotin-conjugated 
goat secondary antibody for $1 \mathrm{hr}$ at room temperature, and visualized with $\mathrm{ABC}-\mathrm{DAB}$ kit (Vector Labs, Burlingame, CA).

\section{Quantitative real-time polymerase chain reaction analysis} Frozen tumor tissue from ERRN and WPERRN mice was homogenized and lysed in Tri-reagent (MRC, Cincinnati, OH). RNAs was isolated from Tri-reagent reagent solutions by chloroform-isopropanol extraction. DNA was prepared from isolated RNAs using High Capacity cDNA Reverse Transcriptase kit (Applied Biosystems, Foster City, CA), per manufacturer instructions. The sequences for primers are as follows: for CCND1 (5'CTC CTC TTC GCA CTT CTG CTC3', and 5'GCG TAC CCT GAC ACC AAT CTC3'), for SMAD3 (5' CCT TCT GGT GCT CCA TCT CC3', and 5'ACA CCT CTC CCA ATG TGT CG3'), for CTSD (5'CCT TTG ACA TCC ACT ACG GC3' and 5'AAG ATG CCA TCA AAC TTG GC3'). Expression of these genes was normalized to expression of $\beta$-glucuronidase (GUS, 5’TTG AGA ACT GGT ATA AGA CGC ATC AG3', 5'TCT GGT ACT CCT CAC TGA ACA TGC3'). Quantitative real-time PCR was performed using SYBR-green in 96-well plates read with 7900 HT Fast Real-Time PCR/Sequence Detection Systems (Applied Biosystems, Foster City, CA).

\section{Cell Based Assays}

For migration assays, $1 \times 10^{5} \mathrm{ER}^{\mathrm{fl} / \mathrm{fl}}$ Ron or $\mathrm{ER}^{-/-}$Ron cells were plated in the top chamber of $0.8 \mu \mathrm{m}$ transwells (Corning Costar Corporation, Cambridge, MA) and were allowed to migrate towards 5\% FBS DMEMF12 media for 24 hours. The number of live cells on the bottom of the transwell was measured using an MTT assay according to the manufacturer's instructions (Sigma-Aldrich, St. Louis, MO) with absorbance read at $570 \mathrm{~nm}$. Invasion assays were performed similar to the migration assays except that the transwell inserts were coated with Matrigel ${ }^{\mathrm{TM}}$ (BD Biosciences, Billerica, MA) and invasion was assessed at $48 \mathrm{hrs}$. For cell death assays, cells were plated at equal densities followed by serum starvation for 48 hours. The cells, including the floating/dead cells, were collected and stained with AnnexinV and propidium iodide (BD Pharmingen, San Diego, CA) and analyzed by flow cytometry according to the manufacturer's instructions. Cell proliferation assays were accomplished by plating cells on glass cover slips. The cells were labeled for 4 hours with 5-bromo-2'-deoxyuridine (BrDU) and stained according to the manufacturer's instructions (RPN20, GE Healthcare Life Sciences, Piscataway, NJ). The number of BrDU positive cells and the total number of cells in a $10 \times$ field were counted and represented as the percentage of BrDU positive cells per group.

\section{Tissue Microarray}

The tissue arrays were purchased from the National Cancer Institute's Cooperative Breast Cancer Tissue Resource (CBCTR 2nd Generation Progression Tissue Microarray). Each array was purchased in duplicate, one serving as an isotype control for the immunohistochemistry that was performed as described above. Samples were scored as previously described for intensity on a 0 3 scale and for percent positivity $(0-100 \%)[22,23]$. The two parameters were multiplied to give an overall score (0-300). Blinded scoring was performed by two independent scorers, and the average score was calculated and used for each sample.

\section{Statistical Analysis}

The statistical test for each experiment is listed in the corresponding figure legend. A p-value of $<0.05$ is considered significant. All data were analyzed using Graphpad Prism software (LaJolla, CA).

\section{Acknowledgements}

We would like to thank Kay-Uwe Wagner and William Muller for providing mice expressing Cre recombinase. This work was supported by Public Health Service Grants CA100002 (S.E.W) and T32CA117846 (A.M.M. and S.E.W.) from the National Institutes of Health, and by grant 1101 BX000803 (S.E.W.) from the Cincinnati Veteran's Administration Medical Center.

\section{Author details}

'Department of Cancer and Cell Biology, University of Cincinnati, College of Medicine, Cincinnati, OH 45267-0521, USA. ²Department of Surgery, University of Cincinnati, College of Medicine, Cincinnati, OH 45267-0521, USA. ${ }^{3}$ Research Service, Cincinnati Veterans Affairs Medical Center, Cincinnati, $\mathrm{OH}$, USA.

\section{Authors' contributions}

AMM and RJM were involved in the design, acquisition and analysis of the data as well as in drafting the manuscript. JKG, DG and KEL were involved in the acquisition of data. SAK was involved in the conception of the studies and the design of reagents for the ERa mouse model. SEW was involved in the conception and design of the study as well as in drafting and revising the manuscript. All authors have read and have approved the final manuscript.

\section{Competing interests}

The authors declare that they have no competing interests.

Received: 23 August 2011 Accepted: 6 January 2012

Published: 6 January 2012

\section{References}

1. Palazzo A, lacovelli $R$, Cortesi E: Past, present and future of targeted therapy in solid tumors. Curr Cancer Drug Targets 2010, 10:433-461.

2. Clarke R, Leonessa F, Welch JN, Skaar TC: Cellular and molecular pharmacology of antiestrogen action and resistance. Pharmacol Rev 2001, 53:25-71.

3. Kato $S$, Endoh $H$, Masuhiro $Y$, Kitamoto T, Uchiyama S, Sasaki H, Masushige S, Gotoh Y, Nishida E, Kawashima H, et al: Activation of the estrogen receptor through phosphorylation by mitogen-activated protein kinase. Science 1995, 270:1491-1494. 
4. McClaine RJ, Marshall AM, Wagh PK, Waltz SE: Ron receptor tyrosine kinase activation confers resistance to tamoxifen in breast cancer cell lines. Neoplasia 2010, 12:650-658.

5. Arpino G, De Angelis C, Giuliano M, Giordano A, Falato C, De Laurentiis M, De Placido S: Molecular mechanism and clinical implications of endocrine therapy resistance in breast cancer. Oncology 2009, 77(Suppl 1):23-37.

6. Britton DJ, Hutcheson IR, Knowlden JM, Barrow D, Giles M, McClelland RA, Gee JM, Nicholson Rl: Bidirectional cross talk between ERalpha and EGFR signalling pathways regulates tamoxifen-resistant growth. Breast Cancer Res Treat 2006, 96:131-146.

7. Meijer D, van Agthoven T, Bosma PT, Nooter K, Dorssers LC: Functional screen for genes responsible for tamoxifen resistance in human breast cancer cells. Mol Cancer Res 2006, 4:379-386.

8. Shou J, Massarweh S, Osborne CK, Wakeling AE, Ali S, Weiss H, Schiff R: Mechanisms of tamoxifen resistance: increased estrogen receptor-HER2/ neu cross-talk in ER/HER2-positive breast cancer. J Natl Cancer Inst 2004, 96:926-935.

9. Wagh PK, Peace BE, Waltz SE: Met-related receptor tyrosine kinase Ron in tumor growth and metastasis. Adv Cancer Res 2008, 100:1-33.

10. Meyer SE, Zinser GM, Stuart WD, Pathrose P, Waltz SE: The Ron receptor tyrosine kinase negatively regulates mammary gland branching morphogenesis. Dev Biol 2009, 333:173-185.

11. Peace BE, Hughes MJ, Degen SJ, Waltz SE: Point mutations and overexpression of Ron induce transformation, tumor formation, and metastasis. Oncogene 2001, 20:6142-6151.

12. Chen $Y Q$, Zhou $Y Q$, Fu LH, Wang D, Wang MH: Multiple pulmonary adenomas in the lung of transgenic mice overexpressing the RON receptor tyrosine kinase. Recepteur d'origine nantais. Carcinogenesis 2002, 23:1811-1819

13. Zinser GM, Leonis MA, Toney K, Pathrose P, Thobe M, Kader SA, Peace BE, Beauman SR, Collins MH, Waltz SE: Mammary-specific Ron receptor overexpression induces highly metastatic mammary tumors associated with beta-catenin activation. Cancer Res 2006, 66:11967-11974.

14. Peace BE, Toney-Earley K, Collins MH, Waltz SE: Ron receptor signaling augments mammary tumor formation and metastasis in a murine model of breast cancer. Cancer Res 2005, 65:1285-1293.

15. Maggiora P, Marchio S, Stella MC, Giai M, Belfiore A, De Bortoli M, Di Renzo MF, Costantino A, Sismondi P, Comoglio PM: Overexpression of the RON gene in human breast carcinoma. Oncogene 1998, 16:2927-2933.

16. Daniel CW, Silberstein GB, Strickland P: Direct action of 17 beta-estradiol on mouse mammary ducts analyzed by sustained release implants and steroid autoradiography. Cancer Res 1987, 47:6052-6057.

17. Fan P, Wang J, Santen RJ, Yue W: Long-term treatment with tamoxifen facilitates translocation of estrogen receptor alpha out of the nucleus and enhances its interaction with EGFR in MCF-7 breast cancer cells. Cancer Res 2007, 67:1352-1360

18. Feng Y, Manka D, Wagner KU, Khan SA: Estrogen receptor-alpha expression in the mammary epithelium is required for ductal and alveolar morphogenesis in mice. Proc Natl Acad Sci USA 2007, 104:14718-14723.

19. Silberstein GB, Van Horn K, Shyamala G, Daniel CW: Essential role of endogenous estrogen in directly stimulating mammary growth demonstrated by implants containing pure antiestrogens. Endocrinology 1994, 134:84-90

20. Minn AJ, Gupta GP, Siegel PM, Bos PD, Shu W, Giri DD, Viale A, Olshen AB, Gerald WL, Massague J: Genes that mediate breast cancer metastasis to lung. Nature 2005, 436:518-524.

21. Sotiriou C, Wirapati P, Loi S, Harris A, Fox S, Smeds J, Nordgren H, Farmer P, Praz V, Haibe-Kains B, et al: Gene expression profiling in breast cancer: understanding the molecular basis of histologic grade to improve prognosis. J Natl Cancer Inst 2006, 98:262-272.

22. Wagh PK, Gray JK, Zinser GM, Vasiliauskas J, James L, Monga SP, Waltz SE: beta-Catenin is required for Ron receptor-induced mammary tumorigenesis. Oncogene 2011.

23. Thobe MN, Gurusamy D, Pathrose P, Waltz SE: The Ron receptor tyrosine kinase positively regulates angiogenic chemokine production in prostate cancer cells. Oncogene 2010, 29:214-226.

24. Torres-Arzayus Ml, Font de Mora J, Yuan J, Vazquez F, Bronson R, Rue M, Sellers WR, Brown M: High tumor incidence and activation of the PI3K/
AKT pathway in transgenic mice define AIB1 as an oncogene. Cancer Cell 2004, 6:263-274

25. Augereau P, Miralles F, Cavailles V, Gaudelet C, Parker M, Rochefort H: Characterization of the proximal estrogen-responsive element of human cathepsin D gene. Mol Endocrinol 1994, 8:693-703.

26. Lin Z, Reierstad S, Huang CC, Bulun SE: Novel estrogen receptor-alpha binding sites and estradiol target genes identified by chromatin immunoprecipitation cloning in breast cancer. Cancer Res 2007, 67:5017-5024.

27. Sabbah M, Courilleau D, Mester J, Redeuilh G: Estrogen induction of the cyclin D1 promoter: involvement of a CAMP response-like element. Proc Natl Acad Sci USA 1999, 96:11217-11222.

28. Leonard GD, Swain SM: Ductal carcinoma in situ, complexities and challenges. J Natl Cancer Inst 2004, 96:906-920.

29. Russo J, Russo $\mathrm{H}$ : The role of estrogen in the initiation of breast cancer. $J$ Steroid Biochem Mol Biol 2006, 102:89-96.

30. Ren Y, Wu L, Frost AR, Grizzle W, Cao X, Wan M: Dual effects of TGF-beta on ERalpha-mediated estrogenic transcriptional activity in breast cancer. Mol Cancer 2009, 8:111.

31. Mizuochi T, Yee ST, Kasai M, Kakiuchi T, Muno D, Kominami E: Both cathepsin B and cathepsin D are necessary for processing of ovalbumin as well as for degradation of class II MHC invariant chain. Immunol Lett 1994, 43:189-193.

32. Liu QP, Fruit K, Ward J, Correll PH: Negative regulation of macrophage activation in response to IFN-gamma and lipopolysaccharide by the STK/RON receptor tyrosine kinase. J Immunol 1999, 163:6606-6613.

33. Nikolaidis NM, Gray JK, Gurusamy D, Fox W, Stuart WD, Huber N, Waltz SE: Ron receptor tyrosine kinase negatively regulates TNFalpha production in alveolar macrophages by inhibiting NF-kappaB activity and Adam17 production. Shock 2010, 33:197-204

34. de la Haba J, Gomez A, Duenas R, Ribelles N, Mendez MJ, Serrano R, Font P, Aranda $E$ : The quotient of number of nodes and tumour size $(N / T)$ from primary breast cancer predicts the clinical course after diagnosis of distant relapse. Eur J Surg Oncol 2004, 30:346-351.

35. Gonzalez-Angulo AM, Morales-Vasquez F, Hortobagyi GN: Overview of resistance to systemic therapy in patients with breast cancer. Adv Exp Med Biol 2007, 608:1-22.

36. Wagner KU, McAllister K, Ward T, Davis B, Wiseman R, Hennighausen L: Spatial and temporal expression of the Cre gene under the control of the MMTV-LTR in different lines of transgenic mice. Transgenic Res 2001, 10:545-553.

37. Wagner KU, Wall RJ, St-Onge L, Gruss P, Wynshaw-Boris A, Garrett L, Li M, Furth PA, Hennighausen $\mathrm{L}$ : Cre-mediated gene deletion in the mammary gland. Nucleic Acids Res 1997, 25:4323-4330.

doi:10.1186/1476-4598-11-2

Cite this article as: Marshall et al: Estrogen receptor alpha deletion enhances the metastatic phenotype of Ron overexpressing mammary tumors in mice. Molecular Cancer 2012 11:2

\section{Submit your next manuscript to BioMed Central and take full advantage of:}

- Convenient online submission

- Thorough peer review

- No space constraints or color figure charges

- Immediate publication on acceptance

- Inclusion in PubMed, CAS, Scopus and Google Scholar

- Research which is freely available for redistribution

Submit your manuscript at www.biomedcentral.com/submit
C Biomed Central 